\title{
Biochemical Genetics of the $\alpha$-Keto Acid Dehydrogenase Complexes of Escherichia coli K12: Isolation and Biochemical Properties of Deletion Mutants
}

\author{
By D. LANGLEY* AND J. R. GUEST \\ Department of Microbiology, Sheffield University, Sheffield SIo $2 T N$
}

(Received 15 October 1976)

\begin{abstract}
SUMMARY
Mutants of Escherichia coli KI2 with deletions in the nadC-lpd region of the chromosome were obtained for use in studies on the expression of the ace (pyruvate dehydrogenase complex, specific components) and lpd (lipoamide dehydrogenase) genes. These were isolated by selecting spontaneous aroP mutants (lacking the general aromatic amino-acid permease and thus resistant to inhibitory aromatic amino-acid analogues) and screening for auxotrophy due to deletions extending into neighbouring genes. From 2892 isolates tested, the AroP- phenotypes of 2322 were confirmed and, of these, 28 stable and independently-derived auxotrophs were designated as deletion mutants.

Six nutritionally-distinct categories were recognized: $\mathrm{Nad}^{-}$(8 strains); $\mathrm{Nad}^{-}$Ace- (7); $\mathrm{Nad}^{-}$'Ace-' (3); Ace- (8); 'Ace-' (I); Lpd- (I). The Ace- phenotypes of four isolates designated 'Ace-' were leaky and enzymological studies confirmed that they had less than $7 \%$ of parental pyruvate dehydrogenase complex activity.

Enzymological studies showed that the ${ }_{15} \mathrm{Ace}^{-}$or $\mathrm{Nad}^{-} \mathrm{Ace}^{-}$strains all lacked the pyruvate dehydrogenase complex and pyruvate dehydrogenase (EIp) activities and only three retained detectable dihydrolipoamide acetyltransferase (E2p). The one $\mathrm{Lpd}^{-}$strain lacked pyruvate dehydrogenase, dihydrolipoamide acetyltransferase and lipoamide dehydrogenase $\left(\mathrm{E}_{3}\right)$ activities as well as the activities of the pyruvate and $\alpha$-ketoglutarate dehydrogenase complexes.

The results confirmed the gene order nadC-aroP-aceE-aceF-lpd and indicated that no other essential functions are determined by genes within the nadC-lpd region. Resistance to lactate during growth of pps mutants on acetate was directly related to the specific activity of the pyruvate dehydrogenase complex. None of the deletions promoted the high degree of resistance characteristically associated with constitutive expression of the dehydrogenase complex. Six pps mutants having Ace ${ }^{+}$ or 'Ace' phenotypes were more sensitive than the parental strains and expression of their ace operons appeared to be affected; most sensitive were the Ace ${ }^{-}$strains which lacked pyruvate dehydrogenase complex and phosphoenolpyruvate synthetase activities.

The lipoamide dehydrogenase activities of the deletion strains ( $\left.\mathrm{Lpd}^{+}\right)$ranged between $30 \%$ and $100 \%$ of parental levels indicating that expression of the lpd gene may be affected by the ace operon but can be independent.
\end{abstract}

\section{INTRODUCTION}

The pyruvate dehydrogenase (EIp) and dihydrolipoamide acetyltransferase (E2p) components of the pyruvate dehydrogenase complex are specified by a pair of closely-

* Present address: Beecham Pharmaceuticals, Clarendon Road, Worthing, West Sussex, BNI4 8QH. 
linked genes, ace $E$ and $a c e F$ respectively, at 2 min on the Escherichia coli KI 2 linkage map (Henning \& Herz, 1964). Mutants lacking pyruvate dehydrogenase complex activity require acetate for aerobic growth on glucose or succinate, but they can grow on unsupplemented acetate medium. The corresponding dehydrogenase (EI kg) and succinyltransferase (E2kg) components of the $\alpha$-ketoglutarate dehydrogenase complex are specified by a second pair of closely-linked genes, $s u c A$ and $s u c B$, at 16 min (Herbert \& Guest, 1969). Mutants lacking $\alpha$-ketoglutarate dehydrogenase complex activity require succinate or a dual supplement of lysine plus methionine for aerobic growth on glucose, and they cannot grow on acetate minimal medium. The lipoamide dehydrogenase $\left(E_{3}\right)$ components of both complexes are specified by a single gene (lpd) which is located very close to the ace $F$ gene at the distal end of the ace operon (Guest \& Creaghan, 1972, 1973; Alwine, Russell \& Murray, 1973; Guest, 1974). The ace and lpd genes are also very close to the nadC (quinolinate phosphoribosyltransferase) and aroP (general aromatic amino-acid permease) genes and the segment of chromosome containing the sequence nadC-aroP-aceE-aceF-lpd corresponds to approximately $0.2 \mathrm{~min}$ or 8 kilobases. Lipoamide dehydrogenase mutants lack the overall activities of both multienzyme complexes and consequently require nutritional supplements of both acetate and succinate for best aerobic growth on glucose. They cannot grow on acetate or succinate minimal media. All ace, suc and lpd mutants can grow anaerobically on unsupplemented glucose medium. Under these conditions the corresponding functions are not essential and other mechanisms for the synthesis of acetate and succinate are induced (or derepressed).

Studies with polar mutants have established that expression of both the ace and suc operons is polarized from the dehydrogenase to acyltransferase genes in a clockwise direction, ace $\overrightarrow{E F}$ and $s u c \overrightarrow{A B}$ relative to the linkage map (Henning et al., I966, I968, 1969; Creaghan \& Guest, 1972). A regulatory element linked to the ace genes and controlling their expression has also been detected (Flatgaard, Hoehn \& Henning, 197I). The existence of a single $l p d$ gene raises the problem of how its expression is controlled relative to the syntheses of the two enzyme complexes, which appear to be independently regulated (Langley \& Guest, unpublished observations). Lipoamide dehydrogenase synthesis is reduced by polar ace mutations and, to a lesser extent, by polar suc mutations, but lpd expression is never less than $30 \%$ of normal in strains combining the most polar ace $E$ and sucA mutations (Creaghan \& Guest, 1972).

Studies with Salmonella typhimurium LT2 indicate that the genes specifying its $\alpha$-keto acid dehydrogenase complexes are organized in the same way as in $E$. coli (Langley \& Guest, 1974). Mutants of $S$. typhimurium analogous to the $a c e E$ and $s u c A$ mutants of $E$. coli have been examined. Two mutants were found which possessed deletions in the ace-lpd region extending to the aroP gene (strain SMI6) and to the nadC plus aroP genes (strain SM5I). Mutation or deletion of the aroP gene affects the transport of aromatic amino acids and thus generates resistance to analogues which would normally inhibit growth (Ames \& Roth, 1968; Brown, 1970).

Strains of $E$. coli with deletions in or near the ace and lpd genes could be useful for studying factors controlling the expression of the ace operon and the lpd gene. The close proximities of the aroP, ace and lpd genes in E. coli (as in S. typhimurium) offered the possibility of isolating such deletion strains by screening spontaneous aroP mutants selected in the presence of appropriate supplements (acetate, succinate and nicotinate) for auxotrophy due to deletions extending into the neighbouring genes. This paper describes the isolation and biochemical properties of 28 auxotrophic aroP deletion strains representing six nutri- 


\section{Table I. Strains of Escherichia coli $\mathbf{K I} 2$}

$\begin{array}{lll}\text { Strain } & \text { Characteristics } & \text { Source } \\ \text { H } & \text { Hfr; thiA, azi } & \text { W. Hayes } \\ \text { KI } & \text { metB, thy, azi } & \text { H. L. Konberg } \\ \text { KI-I } & \text { metB, thy, azi, ton, pps } & \text { H. L. Kornberg } \\ \text { KI-1LR8-16 } & \text { metB, thy, azi, ton, pps, ace } & \text { U. Henning } \\ \text { A2T3 } & \text { aceE2, trpE61 }(\lambda) & \text { U. Henning } \\ \text { A6 } & a c e E 6(\lambda) \text { ? } & \text { U. Henning } \\ \text { AIO } & \text { aceF10 }(\lambda) & \text { U. Henning } \\ \text { WI485AI } & \text { aceE1, supE42 } & \text { J. R. Guest } \\ \text { T3A58lpdI } & \text { trpE61, trpA58, lpd-1 } & \text { J. R. Guest }\end{array}$

* $a c e^{\mathbf{c}}$ denotes the presence of a mutation which leads to constitutive expression of the ace genes.

tionally-distinct groups. A preliminary report of some of this work has been published previously (Langley \& Guest, 1975).

\section{METHODS}

Bacterial strains. The characteristics and sources of the strains of Escherichia coli are listed in Table I. Strain KI and its derivatives, KI-I and KI-ILR8-I6, were originally defined as Hfr and thi but these characteristics had been lost. Strain KI-ILR8-I6 was one of the Ace strains which synthesize the pyruvate dehydrogenase complex constitutively (Flatgaard et al., 1971). The parent for strains A2T3, A6 and AIO is probably Ymel (Henning, Herz \& Szolyvay, 1964). Deletion mutants were isolated in three parental strains, H, KI-I and KI-ILR8-I6, and designated by the prefixes $\mathrm{H} \Delta, \mathrm{K} \Delta$ and $\mathrm{c} \Delta$ (c denoting the constitutive parent), respectively. Each deletion was given a different number and when deletions isolated in strain $\mathrm{H}$ were transferred to the $\mathrm{KI}-\mathrm{I}$ background by conjugation, these strains were designated by the prefix $\mathrm{KH} \Delta$ but retained the original number.

Media. Minimal medium E of Vogel \& Bonner (1956) was used in all experiments. Carbon sources were D-glucose (IO $\mathrm{mM}$ ), potassium acetate $(50 \mathrm{mM})$ or potassium succinate $(50 \mathrm{mM})$. Supplements were added as required: acetate $(2 \mathrm{mM}$ with glucose as substrate, or $4 \mathrm{mM}$ with succinate), succinate $(2 \mathrm{mM})$, L-lysine $\left(40 \mu \mathrm{g} \mathrm{ml}^{-1}\right)$, L-methionine $\left(20 \mu \mathrm{g} \mathrm{ml}^{-1}\right)$, other amino acids $\left(30 \mu \mathrm{g} \mathrm{ml}^{-1}\right)$, vitamins $\left(10 \mu \mathrm{g} \mathrm{ml}^{-1}\right)$ and streptomycin sulphate $(200 \mu \mathrm{g}$ $\mathrm{ml}^{-1}$ ). The complete medium used for routine growth and maintenance was $L$ broth (Lennox, I955). Media were solidified with Bacto-agar ( $\left.5 \mathrm{~g} \mathrm{l}^{-1}\right)$ when required. With Thy ${ }^{-}$ strains, thymine $\left(50 \mu \mathrm{g} \mathrm{ml}^{-1}\right)$ was added to complete and defined media to ensure optimal growth.

Isolation of spontaneous aroP mutants. Independent stationary-phase cultures of the parental strains were prepared by inoculating single colonies into $5 \mathrm{ml} \mathrm{L}$ broth and shaking for $16 \mathrm{~h}$ at $37^{\circ} \mathrm{C}$. The bacteria were washed twice and concentrated ro-fold (to about $5 \times 10^{10}$ organisms $\left.\mathrm{ml}^{-1}\right)$ in sterile saline $\left(9 \mathrm{~g} \mathrm{l}^{-1}\right)$. Samples $(0.05$ and $0.1 \mathrm{ml})$ were plated on a selective glucose minimal medium (Gnaslm-aro) containing supplements of nicotinate, acetate, succinate, lysine, methionine and the analogues DL-5-methyltryptophan $\left(20 \mu \mathrm{g} \mathrm{ml}^{-1}\right)$ and $\beta$-2-thienyl-DL-alanine ( $20 \mu \mathrm{g} \mathrm{ml}^{-1}$ ), plus other specific supplements required by the parental strains. After $48 \mathrm{~h}$ at $37^{\circ} \mathrm{C}, 50$ to 200 resistant colonies appeared on each plate. Some 50 to 150 colonies from each starting culture were picked, diluted in saline and grown as small patches on the same medium (25. to 50 patches per plate). These plates served as master plates for replica-plating to sub-master plates (G-aro), which contained the analogues but not the supplements, and to control plates (Gnaslm-aro). The sub-master plates were also replica-plated immediately to plates $(G)$ which contained neither analogues nor 
supplements, and to control plates (Gnaslm) which lacked the analogues. Examination of all the plates after incubation confirmed the presence of aroP mutants and permitted the detection of auxotrophic aroP strains. The relatively large inoculum provided by the first replica plates was essential for demonstrating the AroP- phenotype. Conversely, the second set of replica plates improved the sensitivity of the method for detecting auxotrophs by testing growth from small inocula in the absence of the amino-acid analogues. Potential auxotrophic aroP mutants were purified on the selective medium (Gnaslm-aro) and details of their growth requirements were elucidated by further tests. Only one mutant of a particular phenotypic class was selected from each independent parental culture.

Reversion tests. Cultures ( $16 \mathrm{~h}$ at $37^{\circ} \mathrm{C}$ in L broth) of mutant organisms were washed twice with saline and duplicate samples containing $10^{8}$ and $10^{9}$ bacteria were plated on appropriate media. The plates were incubated at $37^{\circ} \mathrm{C}$, examined at regular intervals for 5 days and, when present, the number of spontaneous revertant colonies was scored.

Conjugation. Deletions isolated in strain $\mathbf{H}$ were transferred by conjugation to the KI-I (Pps-, $\mathrm{Met}^{-}$) background in order to investigate the regulatory state of the ace operon by lactate-sensitivity tests and to provide a complete set of deletions in one genetic background containing a reference marker (met $\left.B^{-}\right)$for subsequent fine-structure genetic analyses. Overnight $L$ broth cultures of the recipient, $k \Delta \mathrm{I} 8 \mathrm{~s}$, a spontaneous streptomycin-resistant derivative of mutant $\mathrm{K} \Delta \mathrm{I} 8$ ( $\triangle a r o P-l p d$, met $B$, thy, pps; see Results), were diluted ro-fold in fresh $\mathrm{L}$ broth and grown for $90 \mathrm{~min}$ at $37^{\circ} \mathrm{C}$ with shaking. The donor strains (nine stable H $\Delta$ strains, see Results) were grown as stationary slanted $5 \mathrm{ml}$ cultures and likewise were subcultured into fresh $\mathrm{L}$ broth. Equal portions ( $\mathrm{I} \mathrm{ml}$ ) of the exponential-phase cultures containing approximately $2 \times 10^{9}$ donor and $2 \times 10^{8}$ recipient bacteria per ml were mixed gently and incubated at $37^{\circ} \mathrm{C}$ without shaking for $20 \mathrm{~min}$. Mating pairs were separated by vortex mixing for $\mathrm{I}$ min and $\mathrm{Lpd}^{+} \mathrm{Str}^{\mathrm{R}}$ recombinants were selected immediately by plating dilutions in saline on acetate minimal medium plus streptomycin $\left(200 \mu \mathrm{g} \mathrm{ml}^{-1}\right)$ and appropriate supplements, and enriched with nutrient broth (Difco; $2 \mathrm{ml} \mathrm{l}^{-1}$ ). The recombinants from each cross were purified and tested and a series of strains possessing the characteristics of the donor deletion and the recipient $\left(\mathrm{Met}^{-}, \mathrm{Thy}^{-}, \mathrm{Pps}^{-}, \mathrm{Str}^{\mathrm{R}}\right.$ ) were readily isolated. These strains were designated by the prefix $\mathrm{KH} \Delta$ but retained the number of the original $\mathrm{H}$ deletion strain.

Preparation of bacteria-free extracts. Organisms were grown in $500 \mathrm{ml}$ batches of salts medium E, with glucose (Io $\mathrm{mM}$ ) and relevant supplements, in 21 Erlenmeyer flasks shaken at $37^{\circ} \mathrm{C}$. Bacteria were harvested in the late-exponential phase by centrifuging, washed twice, and resuspended in potassium phosphate buffer $(0.04 \mathrm{M}, \mathrm{pH} 7.0$ ) to obtain $250 \mathrm{mg}$ wet wt organisms $\mathrm{ml}^{-1}$. Suspensions were disrupted with an ultrasonic cell disintegrator (M.S.E., $150 \mathrm{~W}$ ) for $4 \mathrm{~min}$ at $0^{\circ} \mathrm{C}$ (two $2 \mathrm{~min}$ treatments with an interval for cooling). The supernatant ultrasonic extracts were obtained after centrifuging ( $24000 \mathrm{~g}$ for $30 \mathrm{~min}$ ), and protein was determined according to Lowry et al. (1951) using crystalline bovine serum albumin as standard. Samples of each mutant culture were tested to ensure that reversion had not occurred.

Enzyme assays. Specific activities [ $\mu \mathrm{mol}$ substrate transformed (mg protein) $)^{-1} \mathrm{~h}^{-1}$ ] were determined in the region of proportionality between initial reaction velocity and protein concentration. All spectrophotometric assays were carried out in $10 \mathrm{~mm}$ light-path silica cuvettes at $25{ }^{\circ} \mathrm{C}$ with a Beckman DB GT double-beam spectrophotometer coupled to a Servoscribe Is potentiometric recorder.

Pyruvate dehydrogenase ( $\mathrm{pdh}$ ) complexand $\alpha$-ketoglutarate dehydrogenase (kgdh) complex were assayed spectrophotometrically at $366 \mathrm{~nm}$ and $\mathrm{pH} 8.5$ by following the $\alpha$-keto acid- 
dependent reduction of 3 -acetylNAD according to Guest and Creaghan (1973); the only modification was the addition of $\mathrm{MgCl}_{2}(5 \mu \mathrm{mol})$ to obtain maximum activity when assaying the pyruvate dehydrogenase complex.

Pyruvate dehydrogenase (EI ; FC I . 2.4.I) and $\alpha$-ketoglutarate dehydrogenase (EI kg; EC I .2.4.2) were assayed spectrophotometrically at $420 \mathrm{~nm}$ and $\mathrm{pH} 6 \cdot 3$ with ferricyanide as the electron acceptor, according to Hager \& Kornberg (196I). As discussed by Langley \& Guest (1974), the assay is not specific for Erp as the flavoprotein pyruvate oxidase (EC $I .2 .2 .2$ ), which is present in extracts in varying amounts, is also active in this assay.

Lipoamide dehydrogenase (E3; EC I .6.4.3) was assayed spectrophotometrically at 366 $\mathrm{nm}$ and $\mathrm{pH} 7.8$ by recording the dihydrolipoate-dependent reduction of 3-acetylNAD according to Creaghan \& Guest (1972).

Dihydrolipoamide acetyltransferase (E2p; EC 2.3.I.I2) was assayed at $30^{\circ} \mathrm{C}$ and $\mathrm{pH} 7 \cdot 0$ by the method of Willms et al. (1967) which measures the production of the heat-stable thioester $S$-acetyldihydrolipoamide from dihydrolipoate and acetyl-CoA (generated from acetylphosphate and $\mathrm{CoA}$ with phosphotransacetylase). Reaction mixtures containing I to $2 \mathrm{mg}$ protein were incubated for 5 to $10 \mathrm{~min}$ and the product was determined by the hydroxamate procedure of Lipmann \& Tuttle (1945). Wild-type and constitutive rates were readily detected but the method lacked the sensitivity to detect with any degree of certainty specific activities of less than I (i.e. less than $15 \%$ of the wild type).

More sensitive tests for the presence of acetyltransferase (E2p) and positive identifications of which component(s) of the dehydrogenase complexes were missing in specific mutants were achieved by complementation assays. Overall $\alpha$-keto acid dehydrogenase complex activities could be reconstituted by mixing extracts of mutants which lack specific components or by adding purified lipoamide dehydrogenase to extracts of $l p d$ mutants. Details of the complementation assays are described by Guest \& Creaghan (1973).

Materials. Coenzyme A, acetylphosphate (potassium-lithium salt), 3-acetylNAD and phosphotransacetylase were obtained from Boehringer; DL-lactate (lithium salt), $\alpha$-ketoglutarate (monosodium salt), pyruvate (sodium salt), thiamin pyrophosphate and $\beta-2-$ thienyl-DL-alanine were from Sigma; and $\alpha$-lipoic acid (DL-6,8-thioctic acid) and DL-5methyltryptophan were from Koch-Light. DL-Dihydro- $\alpha$-lipoic acid was prepared by the method of Gunsalus \& Razzell (1957). Purified pig heart lipoamide dehydrogenase was from Miles-Seravac, Maidenhead, Berkshire, and a sample of the same enzyme from $E$. coli B was kindly provided by $\mathrm{Dr}$ C. H. Williams, Jr (Department of Biochemistry, University of Michigan, Ann Arbor, Michigan, U.S.A.).

\section{RESULTS}

\section{Isolation of potential deletion strains amongst spontaneous aroP mutants}

The close proximity of the general amino-acid permease gene (aroP) to the genes determining the pyruvate dehydrogenase complex (aceE, aceF and lpd) in Escherichia coli KI2 (Guest, 1974), and the existence of two strains of Salmonella typhimurium LT2 deleted for these genes (Langley \& Guest, 1974), suggested that comparable strains with deletions extending into neighbouring genes may be found among spontaneous aroP mutants of $E$. coli. Mutants (aroP) defective in the general aromatic amino-acid permease are characterized by their resistance to inhibitory analogues such as $p$-fluorophenylalanine, $\beta$-2-thienylalanine and 5-methyltryptophan. Different combinations of these analogues were tested over a range of concentrations to determine optimum selective conditions. The combination of at least two analogues ensured that only aroP mutants survived, because resistance to single 


\section{Table 2. Isolation of spontaneous aroP mutants and the detection of potential deletion strains as auxotrophs}

Spontaneous mutants resistant to DL-5-methyltryptophan and $\beta$-2-thienyl-DL-alanine were isolated and screened for auxotrophy, as described in Methods. The results include four independent aroP auxotrophs of strain $\boldsymbol{H}$ which were later found not to be deletion mutants.

\begin{tabular}{lccccc}
\multicolumn{1}{c}{ Strain } & $\begin{array}{c}\text { Independent } \\
\text { cultures }\end{array}$ & $\begin{array}{c}\text { Colonies } \\
\text { tested }\end{array}$ & $\begin{array}{c}\text { Confirmed } \\
\text { aroP mutants }\end{array}$ & $\begin{array}{c}\text { Auxotrophic } \\
\text { aroP } \text { mutants }\end{array}$ & $\begin{array}{c}\text { Independent } \\
\text { isolates }\end{array}$ \\
H & 15 & 1037 & 969 & 25 & 13 \\
KI-I & 15 & 940 & 612 & 27 & 9 \\
KI-ILR8-16 & 12 & 915 & 741 & 13 & 10 \\
Totals & 14 & 915 & 2322 & 65 & 32
\end{tabular}

analogues could be due to mutations affecting the regulation of amino-acid biosynthesis or specific transport systems. Spontaneous aroP mutants were accordingly selected on appropriate glucose minimal media supplemented with 5 -methyltryptophan plus $\beta$-2-thienylalanine and nicotinate, acetate, succinate, lysine and methionine, which may be required as a consequence of deleting genes adjacent to aroP (see Methods). Three parental strains, $\mathbf{H}$, KI-I (Pps ${ }^{-}$), KI-ILR8-I6 (Pps $^{-}$, Ace $^{\mathrm{c}}$ ), were chosen as the most suitable for studying the effects of deletion on the expression of the ace operon and the regulation of the lpd gene.

Potential aroP mutants appeared at a frequency of approximately $I$ per $3 \times 10^{7}$ bacteria plated. However, the actual frequency may be lower because it is likely that, after plating, several generations occur before growth is inhibited. The results of $4 \mathrm{I}$ independent experiments are summarized in Table 2. Of the 2892 potential AroP- colonies tested, $80 \%$ retained their resistance to the inhibitory analogues. The remaining $20 \%$ failed to grow when transferred to the same medium on which they had been selected. Growth of these isolates during the initial selection may have been due to neighbouring AroP- $^{-}$colonies excreting aromatic amino acids which neutralized the effects of one or both analogues. Of the confirmed aroP mutants, 65 (i.e. $2.8 \%$ ) were auxotrophs, which could have arisen by deletion of neighbouring genes (Table 2), and these were recovered from 28 of the $4 \mathrm{I}$ starting cultures.

\section{Nutritional classification of the auxotrophic aroP mutants}

Using a variety of different media, tests with the 65 auxotrophic aroP mutants indicated the presence of six nutritionally-distinct classes (Table 3). Only one representative of a particular phenotypic class was retained from each starting culture so that a total of 32 independently-derived auxotrophic aroP mutants were chosen for further study (Table 2). The number of independent isolates in each case is included in Table 3. Eight independent isolates were $\mathrm{Nad}^{-}$, requiring a single supplement of nicotinate for growth on glucose, succinate and acetate media (Table 3). The strains exhibited a typical Ace- phenotype by requiring acetate for growth on glucose and succinate media and being able to grow on acetate minimal medium (Table 3). A further mutant required acetate for growth on succinate but the acetate supplement was not essential for growth on glucose although it did improve the rate and extent of growth. This appeared to represent a distinct class of Acelesion which was designated 'Ace-' to signify the leaky or partial nature of the phenotype (Table 3). Eight independent isolates with a composite $\mathrm{Nad}^{-} \mathrm{Ace}^{-}$phenotype required nicotinate and acetate for growth on glucose and succinate media and needed nicotinate for growth on acetate. The acetate requirement of a further three nicotinate-dependent strains was less apparent on succinate as well as glucose media and these were placed in a separate 


\section{Table 3. Nutritional classes of auxotrophic aroP mutants}

The nutritional requirements of 65 auxotrophic aroP mutants were investigated by streaking dilute suspensions in saline on the test media shown below. The six nutritional classes are based on the corresponding growth responses. The number of independent isolates representing each class is also recorded. Growth was scored after $48 \mathrm{~h}$ at $37^{\circ} \mathrm{C}$ as: ++ , very good; +, good; \pm , poor; - , none. Abbreviations for media (substrates in capital letters, supplements in lower case letters) are: $G$, glucose; $A / a$, acetate; $S / s$, succinate; $n$, nicotinate; aro, 5-methyltryptophan plus $\beta$-2-thienylalanine. All media included relevant supplements for the parental strains.

\begin{tabular}{|c|c|c|c|c|c|c|}
\hline Test medium & $\mathrm{Nad}^{-}$ & $\mathrm{Nad}^{-}{ }^{-} \mathrm{Ace}^{-}$' & $\mathrm{Nad}^{-}$Ace $^{-}$ & 'Ace' & $\mathrm{Ace}^{-}$ & $\mathrm{Lpd}^{-}$ \\
\hline G & - & - & - & + & - & - \\
\hline Gn & ++ & + & - & + & - & - \\
\hline $\mathrm{Ga}$ & - & - & - & ++ & ++ & - \\
\hline Gna & ++ & ++ & ++ & $+t$ & ++ & - \\
\hline Gs & - & - & - & + & - & - \\
\hline Gas & - & - & - & ++ & ++ & ++ \\
\hline Gnas & $+t$ & ++ & ++ & ++ & ++ & ++ \\
\hline Gnas-aro & + & + & + & + & + & + \\
\hline $\mathbf{S}$ & - & - & - & \pm & - & - \\
\hline Sn & - & \pm & - & \pm & - & - \\
\hline $\mathrm{Sa}$ & - & $=$ & - & ++ & $+t$ & - \\
\hline Sna & ++ & ++ & $+t$ & ++ & ++ & - \\
\hline A & - & - & - & + & + & - \\
\hline An & + & + & + & + & + & - \\
\hline $\begin{array}{l}\text { No. of independent } \\
\text { isolates }\end{array}$ & 8 & 3 & 8 & I & 10 & 2 \\
\hline
\end{tabular}

class designated $\mathrm{Nad}^{-}$'Ace-' (Table 3). Only two strains exhibited an Lpd- phenotype, requiring acetate plus succinate for growth on glucose and failing to grow on acetate and succinate media (Table 3). These two strains could combine Ace and $\mathrm{Lpd}^{-}$phenotypes but the Ace- nutritional phenotype is included in, and would be masked by, the $\mathrm{Lpd}^{-}$nutritional phenotype.

\section{Further properties of the auxotrophic aroP mutants}

Stability. An important characteristic of most deletion mutants is their stability; failure to detect reversion is often considered a useful criterion for classifying mutations as deletions and for discriminating between deletions and pleiotropic or multiple mutations. Most point mutations revert spontaneously at frequencies similar to a spontaneous mutation rate of approximately I in $10^{7}$ to $10^{9}$ whilst deletions exhibit reversion frequencies of less than $I$ in I0 ${ }^{10}$ (Demerec, 1960).

The 32 auxotrophic aroP strains were tested for spontaneous reversion to prototrophy on the relevant media (listed in Table 3). Where an auxotroph had more than one nutritional phenotype, the stabilities of the characteristics were tested singly and together, e.g. the $\mathrm{Nad}^{-}$ Ace- strains were tested for reversion to $\mathrm{Nad}^{+} \mathrm{Ace}^{-}, \mathrm{Nad}^{-} \mathrm{Ace}^{+}$and $\mathrm{Nad}^{+} \mathrm{Ace}^{+}$phenotypes. Allowing for four generations of bacterial growth after plating, the tests could detect reversion frequencies of $\mathrm{I}$ in $10^{10}$ or lower. These tests showed that the Ace- phenotypes of three strains $\left(\mathrm{H} \Delta_{7}, \mathrm{H} \Delta 8\right.$ and $\left.\mathrm{H} \Delta 9\right)$ and the $\mathrm{Lpd}^{-}$phenotype of one strain ( $\left.\mathrm{H} \Delta \mathrm{I} 2\right)$ had estimated reversion frequencies greater than $\mathrm{I}$ in $3 \times 10^{7}$, which were higher than the rates obtained with representative ace (A2T3, AI0) and lpd (T3A58lpdr) mutants. These strains were presumed to combine aroP mutations (including deletions) with mutations in the ace ( $\mathrm{H} \Delta_{7}$, $\mathrm{H} \Delta 9)$ or $l p d(\mathrm{H} \Delta \mathrm{I} 2)$ genes and a deletion (nadC-aroP) with an ace point mutation $(\mathrm{H} \Delta 8)$. One 


\section{Table 4. The auxotrophic aroP deletion mutants}

The 28 independently-isolated aroP mutants exhibiting stable auxotrophy are listed in the six phenotypic classes. Each strain is prefixed to denote its parent strain, H, K (KI-I) or C (KI-ILR8-I6, the constitutive strain), followed by $\Delta$ designating the deletion and an individual number given to each potential deletion strain. The dotted line in the aroP-ace region indicates that the deletion may not extend into the ace genes but their expression is affected.

\begin{tabular}{|c|c|c|c|}
\hline Phenotypic class* & Strain designations & $\begin{array}{l}\text { Extent of deletion } \\
\text { nadC-aroP-ace-lpd }\end{array}$ & $\begin{array}{l}\text { deletion } \\
\text { strains }\end{array}$ \\
\hline $\mathrm{Nad}^{-}$ & $\begin{array}{c}\mathrm{H}_{1}, \mathrm{H} \Delta_{2}, \mathrm{H} \Delta_{4}, \mathrm{H} \Delta_{5}, \mathrm{H} \Delta_{6}, \\
\mathrm{H} \Delta_{14}, \mathbf{k} \Delta_{27}, \mathrm{c} \Delta_{44}\end{array}$ & $\longrightarrow$ & 8 \\
\hline $\mathrm{Nad}^{-}{ }^{\prime} \mathrm{Ace}^{-'}$ & $\mathrm{H} \Delta_{3}, \mathrm{c} \Delta_{35}, \mathrm{c} \Delta_{39}$ & $\longrightarrow \cdots$ & 3 \\
\hline $\mathrm{Nad}^{-} \mathrm{Ace}^{-}$ & $\begin{array}{l}\text { н } \Delta_{10}, \mathrm{k} \Delta \mathrm{I} 7, \mathrm{k} \Delta_{22} \dagger, \mathrm{k} \Delta_{32} \\
\mathrm{k} \Delta_{34}, \mathrm{c} \Delta_{42} \dagger, \mathrm{c} \Delta_{46}\end{array}$ & $\longrightarrow$ & 7 \\
\hline 'Ace' & $\mathrm{c} \Delta 47$ & $-\cdots \cdots$ & $\mathbf{I}$ \\
\hline $\mathrm{Ace}^{-}$ & $\begin{array}{l}\text { н } \Delta_{13}, \text { н } \Delta_{15}, \mathrm{k} \Delta_{21}, \mathrm{~K} \Delta_{33} \\
\mathrm{c} \Delta_{37}, \mathrm{c} \Delta_{40}, \mathrm{c} \Delta_{41} \dagger, \mathrm{c} \Delta_{45}\end{array}$ & $\longrightarrow$ & 8 \\
\hline Lpd- $^{-}$ & $\mathrm{K} \Delta \mathrm{I} 8$ & $\longrightarrow$ & I \\
\hline
\end{tabular}

other unstable strain, found among the auxotrophic aroP mutants but not included in the foregoing results, required succinate for growth on glucose, grew poorly on succinate media and failed to grow on acetate. This strain ( $\mathrm{H} \Delta \mathrm{II}$ ) was probably a spontaneous double mutant ( $a r o P, s u c$ ). The remaining 28 strains were stable and it was tentatively concluded that they represent spontaneous aroP deletion mutants and that their auxotrophy is due to extension of the deletions into neighbouring genes. The presumptive deletion mutants are listed in Table 4 according to their phenotypic classes. The range of nutritional phenotypes and the relative abundance of the fundamental classes $\left(\mathrm{Nad}^{-}, \mathrm{Nad}^{-} \mathrm{Ace}^{-}, \mathrm{Ace}^{-}, \mathrm{Lpd}^{-}\right)$ confirms the gene order nadC-aroP-ace-lpd, and the extents of the individual classes of deletion can be approximated as shown in Table 4 . If strain $\mathrm{K} \Delta \mathrm{I} 8$ is assumed to be a deletion strain, its $\mathrm{Lpd}^{-}$nutritional phenotype must be masking an Ace- phenotype in order to form a series consistent with the other classes of deletion.

Streptomycin resistance. One of the characteristics of the $S$. typhimurium deletion strains (SMI6 and SM5I) was resistance to low concentrations $\left(20 \mu \mathrm{g} \mathrm{ml}^{-1}\right)$ of streptomycin (CarrilloCastaneda \& Ortega, 1970; Langley \& Guest, 1974). None of the auxotrophic aroP strains of $E$. coli were resistant to this concentration of streptomycin. The phenomenon appears to be a special feature of the $S$. typhimurium deletion strains and it could be due to the deletion of a specific gene in this region of the $S$. typhimurium chromosome or the combined effects of the deletions and some other mutation present in SMI 6 and SM5I.

Regulatory state of the ace operon. Growth on acetate of mutants (pps) lacking phosphoenolpyruvate synthetase is inhibited by low concentrations of pyruvate or lactate (Brice \& Kornberg, 1967). This is because pyruvate represses the glyoxylate cycle and, whereas wild-type strains can remove the pyruvate with phosphoenolypyruvate synthetase, this is not possible in the mutants. However, the pps mutants can become resistant to pyruvate (or lactate) by further mutations which cause (i) constitutive synthesis of the glyoxylate cycle enzymes (iclR; Kornberg, 1966), (ii) defects in pyruvate or lactate transport (e.g. usp; Kornberg \& Smith, 1967) or (iii) constitutive synthesis of the pyruvate dehydrogenase complex (ace $^{\mathrm{c}}$; Flatgaard et al., 1971). To determine whether the regulation of pyruvate dehydro- 
genase complex synthesis was altered by the aroP deletions, the deletion strains were tested for lactate-resistance on acetate (except the Lpd- strain, which was unable to grow on acetate, and the unstable strains presumed not to be deletion strains). Direct tests were possible with the derivatives of strains KI-I and KI-ILR8-I6 because they already possessed the pps mutation. The deletions in strain $\mathrm{H}\left(\mathrm{Pps}^{+}\right)$were all transferred to the $\mathrm{KI}-\mathrm{I}\left(\mathrm{Pps}^{-}\right)$ background by conjugation with a streptomycin-resistant derivative of strain $\mathrm{K} \Delta \mathrm{I} 8$ (K $\mathrm{K} \mathrm{I} 8 \mathrm{~s})$ to yield a series of streptomycin-resistant deletion strains designated KH $\Delta$. Preliminary tests with the strain $\mathrm{H}$ derivatives $\left(\mathrm{Pps}^{+}\right)$indicated that they were all lactate-resistant despite the fact that several lacked the pyruvate dehydrogenase complex and could be deficient in the ability to remove the inhibitor.

All the Pps- deletion and parental (KI-I and KI-ILR8-I6) strains plus the Pps $^{+}$strain KI, were tested at $37^{\circ} \mathrm{C}$ by streaking dilute suspensions on acetate minimal medium supplemented with lithium DL-lactate at concentrations of $0,5,10,15,30$ and $50 \mu \mathrm{M}$. If a strain was sensitive to lactate then the formation of single colonies was prevented. Strain $\mathrm{KI}\left(\mathrm{Pps}^{+}\right)$ was completely unaffected by $50 \mu \mathrm{M}$-lactate, whereas $\mathrm{KI}-\mathrm{I}\left(\mathrm{Pps}^{-}\right)$tolerated I $5 \mu \mathrm{M}$ but not $30 \mu \mathrm{M}$-lactate and strain KI-ILR8-I6 (Pps-, Ace ${ }^{\mathrm{c}}$ ), constitutive for the pyruvate dehydrogenase complex, grew with $30 \mu \mathrm{M}$ but not $50 \mu \mathrm{M}$-lactate. None of the deletion strains (Pps-, $\mathrm{K} \Delta, \mathrm{KH} \Delta$ and $\mathrm{C} \Delta$ ) were resistant like the constitutive strain (KI-ILR8-I6) and seven of the eight $\mathrm{Nad}^{-} \mathrm{Ace}^{+}$deletion strains exhibited the same sensitivity as $\mathrm{KI}-\mathrm{I}$. The other $\mathrm{Nad}^{+} \mathrm{Ace}^{+}$ deletion strain $(\mathrm{K} \Delta 27)$ was more sensitive than its parent, being inhibited by $15 \mu \mathrm{M}$-lactate; a possible explanation for this could be a lower expression of the ace operon. Strain $c \Delta 44$, the only $\mathrm{Nad}^{-} \mathrm{Ace}^{+}$derivative of KI-ILR8-I6, was also interesting because by resembling KI-I it had lost the ability to tolerate the higher concentrations of lactate characteristic of its parent. The 'Ace-' strains were also more sensitive than the parental strains, being inhibited by $15 \mu \mathrm{M}$-lactate, and this is consistent with impaired expression of the ace operon. Most sensitive were the deletion strains possessing an Ace ${ }^{-}$phenotype. They were inhibited by $10 \mu \mathrm{M}$-lactate and their sensitivity is presumably due to the absence of both pps-and acemediated routes of pyruvate metabolism. Clearly none of the deletions produced lactateresistance corresponding to an $\mathrm{Ace}^{\mathrm{c}}$ phenotype and direct attempts to select such strains on acetate minimal medium plus nicotinate, the aromatic amino-acid analogues and DL-lactate $(30 \mu \mathrm{M})$ were unsuccessful. Higher concentrations of lactate were required for inhibition than those reported by Flatgaard et al. (197I). The discrepancy may be due to the fact that the earlier tests were performed at $42^{\circ} \mathrm{C}$ because the pps mutation is probably leaky at $37^{\circ} \mathrm{C}$. Unfortunately, the strain of KI-I used in the present studies grew very poorly on acetate at $42{ }^{\circ} \mathrm{C}$ and the tests had to be performed at $37^{\circ} \mathrm{C}$.

\section{Enzymological studies}

Ultrasonic extracts of all the auxotrophic aroP mutants were assayed for the pyruvate dehydrogenase (pdh) and $\alpha$-ketoglutarate dehydrogenase (kgdh) complexes and their components EIp, E2p, E3 and EIkg in order to investigate the enzymological phenotype and the effects of the deletions on expression of the ace and lpd genes. The results are summarized in Table 5 .

Parent strains. The parental strains $\mathrm{H}$ and $\mathrm{KI}$-I had very similar activities for all the enzymes tested. Furthermore, the results obtained with strain KI, the Pps ${ }^{+}$parent of KI-I, were also similar indicating that the pps mutation had no effect on these enzymes under the conditions used. The third parental strain (KI-ILR8-I6) had approximately twice as much pdh complex activity as strain $\mathrm{KI}-\mathrm{I}$ (Table 5) and this is in good agreement with the ratio 60:25 reported by Flatgaard et al. (1971) for the pdh complex activities of the same strains grown on glucose. 
Table 5. Specific activities of the a-keto acid dehydrogenase complexes and components in parental and auxotrophic aroP deletion strains

Ultrasonic extracts of cultures grown on glucose medium (supplemented as required) were prepared and the enzymes were assayed as described in Methods. The average activities for determinations with at least two different extracts are quoted. The results quoted for some strains of each phenotypic class are representative of all the other strains not listed but of the same category (see text). Specific activities are expressed as $\mu \mathrm{mol}$ substrate transformed ( $\mathrm{mg}$ protein $)^{-1} \mathrm{~h}^{-1}$. Abbreviations: pdh complex, overall pyruvate dehydrogenase complex; EIp, pyruvate dehydrogenase plus oxidase; E2p, dihydrolipoamide acetyltransferase; E3, lipoamide dehydrogenase; kgdh complex, $\alpha$-ketoglutarate dehydrogenase complex; EI kg, $\alpha$-ketoglutarate dehydrogenase.

\begin{tabular}{|c|c|c|c|c|c|c|c|}
\hline \multirow[b]{2}{*}{ Strain } & \multirow{2}{*}{$\begin{array}{l}\text { Relevant } \\
\text { phenotype }\end{array}$} & \multicolumn{6}{|c|}{ Specinc activity } \\
\hline & & pdh complex & EIp & E2p & $\mathrm{E}_{3}$ & kgdh complex & EIkg \\
\hline $\mathbf{H}$ & $\mathrm{Ace}^{+}$ & $5 \cdot 98$ & $I \cdot 7 I$ & $5 \cdot 90$ & $2 \cdot 07$ & $2 \cdot 50$ & $4 \cdot 85$ \\
\hline KI & Ace $^{+}$ & $4 \cdot 60$ & $x \cdot 80$ & $6 \cdot 20$ & $2 \cdot 25$ & $3 \cdot 10$ & $5 \cdot 40$ \\
\hline KI-I & Ace $^{+}$Pps $^{-}$ & $5 \cdot 00$ & $2 \cdot 0 I$ & $6 \cdot 10$ & $2 \cdot 49$ & $2 \cdot 09$ & $4 \cdot 30$ \\
\hline KI-ILR8-I 6 & $\mathrm{Ace}^{c} \mathrm{Pps}^{-}$ & 10.22 & $3 \cdot 33$ & 13.80 & 3.90 & 3.00 & 3.04 \\
\hline $\mathbf{H} \Delta_{4}$ & $\mathrm{Nad}^{-} \mathrm{Ace}^{+}$ & $4 \cdot 20$ & 2.02 & $3 \cdot 70$ & $2 \cdot 08$ & 3.07 & 4.51 \\
\hline $\mathbf{K} \Delta \mathbf{2 7}$ & $\mathrm{Nad}^{-} \mathrm{Ace}^{+}$ & $I \cdot 52$ & 0.92 & $2 \cdot 30$ & $I \cdot 63$ & $1 \cdot 66$ & $3 \cdot 3 I$ \\
\hline$c \Delta 44$ & $\mathrm{Nad}^{-} \mathrm{Ace}^{+}$ & $4 \cdot 13$ & $2 \cdot 27$ & $6 \cdot 10$ & $3 \cdot 80$ & $2 \cdot 14$ & $2 \cdot 12$ \\
\hline$c \Delta 39$ & $\mathrm{Nad}^{-} \cdot \mathrm{Ace}^{-}$ & 0.36 & 0.37 & 1.60 & $1 \cdot 98$ & $2 \cdot 32$ & $5 \cdot 14$ \\
\hline$c \Delta 47$ & 'Ace' & 0.33 & 0.45 & $1 \cdot 60$ & $1 \cdot 47$ & $I \cdot 55$ & 3.65 \\
\hline $\mathrm{K} \Delta \mathbf{3 3}$ & $\mathbf{A c e}^{-}$ & $<0.01$ & $<0.01$ & $<1.00$ & 0.85 & $I \cdot 84$ & $2 \cdot 40$ \\
\hline$c \Delta 42$ & $\mathrm{Nad}^{-} \mathrm{Ace}^{-}$ & $<0.01$ & $<0.01$ & $<1.00$ & $1 \cdot 56$ & $I \cdot 78$ & 2.88 \\
\hline $\mathrm{k} \Delta \mathrm{I} 8$ & Lpd- $^{-}$ & $<0.01$ & $<0.01$ & $<1.00$ & $<0.01$ & $<0.01$ & $5 \cdot 04$ \\
\hline
\end{tabular}

The activities for E1p, E2p and E3 were also correspondingly higher in the constitutive strains.

Ace $^{+}$deletion strains. Of the eight deletion mutants which were nutritionally Ace $^{+}$ (i.e. all the members of the $\mathrm{Nad}^{-}$class in Table 4), the enzyme activities of six, represented by strain $\mathrm{H}_{4}$ in Table 5, were almost unaffected. One strain $\mathrm{K} \Delta 27$ had only $30 \%$ of the parental pdh complex activity, approximately $50 \%$ of the EIp and $\mathrm{E}_{2}$ components and a reduced $\mathrm{E}_{3}$ activity (Table 5). Although this strain was acetate-independent, the deletion clearly affected expression of the ace operon and this is consistent with the increased lactate-sensitivity of strain $\mathrm{K} \Delta 27$. The remaining $\mathrm{Nad}^{-}$deletion strain, $\mathrm{c} \Delta 44$, no longer expressed the ace operon at the constitutive level (Table 5) and this again had been indicated by its lactate-sensitivity. Presumably the deletion in strain $\mathrm{c} \Delta 44$ removes a regulatory element or fuses the ace operon to another, less efficient, promoter. The $\mathrm{E}_{3}$ activities of the $\mathrm{Ace}^{+}$strains were between $67 \%$ and $100 \%$ of the corresponding parental strains.

'Ace-' deletion strains. The four strains possessing the leaky 'Ace-' phenotype, represented by strains $\mathrm{c} \Delta 39$ and $\mathrm{c} \Delta 47$ in Table 5 , had only about $7 \%$ of the pdh complex activity of the inducible strain KI-I. The EIP and E2p components were also considerably reduced. The $\mathrm{E}_{3}$ activities were affected but less dramatically; between $38 \%$ and $56 \%$ of the parental activity was retained. Again, these deletions appear to have removed one or more control elements or fused the ace structural genes to a promoter with very low efficiency. However, it is interesting that despite the low pdh complex activities, these strains grow on glucose without added acetate but the activities fail to support good growth on unsupplemented succinate medium.

Ace- deletion strains. None of the 15 stable deletions which were nutritionally Ace(eight $\mathrm{Ace}^{-}$and seven $\mathrm{Nad}^{-} \mathrm{Ace}^{-}$), represented by strains $\mathrm{K} \Delta 33$ and $\mathrm{c} \Delta 42$ in Table 5 , possessed detectable pdh complex activity. Seven of the strains gave small positive results in 


\section{Table 6. Complementation between extracts of $\mathrm{Ace}^{-}$and $\mathrm{Lpd}^{-}$ deletion strains and an aceF mutant}

The pyruvate dehydrogenase (pdh) complex activities of mixtures of ultrasonic extracts containing I mg protein of the test strain and an equal amount of the aceF mutant (strain $\mathrm{AIO}$ ) in $\mathrm{I} \mathrm{ml}$ reaction mixtures were assayed. Activities are expressed as total activity in the samples (in $\mu \mathrm{mol} \mathrm{h}^{-1}$ ) after subtracting the very small activities of both participating extracts tested separately.

\begin{tabular}{|c|c|c|}
\hline Test strain & Phenotype & $\begin{array}{c}\text { Activity of pdh } \\
\text { complex }\left(\mu \mathrm{mol} \mathrm{h}^{-1}\right)\end{array}$ \\
\hline $\begin{array}{l}\text { AIO } \\
\text { A2T3 } \\
\text { A6 } \\
\text { WI485AI }\end{array}$ & $\begin{array}{l}\text { AceF- }^{-} \\
\text {AceE- }^{-} \\
\text {AceE }^{-} \\
\text {AceE-- }^{-}\end{array}$ & $\begin{array}{r}<0.01 \\
2.26 \\
0.42 \\
2.42\end{array}$ \\
\hline $\begin{array}{l}H \Delta \Delta_{13} \\
K \Delta 33 \\
C \Delta 46\end{array}$ & $\begin{array}{l}\text { Ace }^{-} \\
\text {Ace }^{-} \\
\text {Nad- Ace }^{-}\end{array}$ & $\begin{array}{l}<0.01 \\
<0.01 \\
<0.01\end{array}$ \\
\hline $\begin{array}{l}\mathrm{x} \Delta \mathbf{2 2} \\
\mathrm{C} \Delta \mathbf{4 1} \\
\mathrm{C} \Delta \mathbf{4 2}\end{array}$ & $\begin{array}{l}\mathbf{N a d}^{-} \mathbf{A c e}^{-} \\
\text {Ace }^{-} \\
\mathbf{N a d}^{-} \mathbf{A c e}^{-}\end{array}$ & $\begin{array}{l}0.53 \\
0.76 \\
0.44\end{array}$ \\
\hline $\mathrm{K} \Delta \mathrm{I} \mathbf{8}$ & Lpd- & $<0.01$ \\
\hline
\end{tabular}

the EIp assay which could be due to pyruvate oxidase but none possessed E2p activity when assayed by the direct method. However, it was difficult to discriminate between the low E2p activities of the leaky 'Ace-' strains (which exhibit weak pdh complex activities) and would be expected to possess E2p) and zero activity which would be predicted for a few Acestrains. The complementation assay proved more sensitive for detecting the presence of the active E2p component. Extracts of each Ace $^{-}$deletion strain were mixed with an extract of strain AIO (an $a c e F$ mutant which contains Erp and $\mathrm{E}_{3}$ components but no E2p) and active E2p was detected by its ability to reconstitute overall pdh complex activity. By this criterion, three deletion strains with Ace ${ }^{-}$phenotypes $(\mathrm{K} \Delta 22, \mathrm{c} \Delta 4 \mathrm{I}$ and $\mathrm{c} \Delta 42)$ complemented strain AIo (Table 6) and it was concluded that they retain intact and functional ace $F$ genes. No complementation was detected with the 12 remaining Ace- strains represented by strains $\mathrm{H} \Delta \mathrm{I} 3, \mathrm{~K} \Delta 33$ or $\mathrm{C}_{4} 46$ in Table 6 . All the Ace- strains contained lipoamide dehydrogenase (E3) at between $30 \%$ and $95 \%$ of the parental level and they all possessed the activities of the $\alpha$-ketoglutarate dehydrogenase complex and its dehydrogenase component EIkg.

Lpd- deletion strain. The one stable strain designated $\mathrm{Lpd}^{-}$by nutritional criteria $(\mathrm{K} \Delta \mathrm{r} 8)$ had no detectable E3, pdh or kgdh complex activities, although EIkg was present (Table 5). Furthermore, strain $\mathrm{k} \Delta \mathrm{I} 8$ lacked EIp (Table 5) and no E2p activity could be detected either by direct or complementation tests (Tables 5 and 6). This confirms that the Lpdnutritional phenotype is masking the Ace- phenotype. Complementation studies were also performed by adding graded amounts of purified lipoamide dehydrogenase from $E$. coli $B$ and pig heart to extracts of strain $\mathrm{K} \Delta \mathrm{I} 8$ in studies similar to those with lpd mutants of $E$. coli and the deletion strains of $S$. typhimurium (Guest \& Creaghan, 1973; Langley \& Guest, I974). Overall kgdh complex activity was restored, but no pdh complex activity could be recovered with either source of the $\mathrm{E}_{3}$ component. It may thus be concluded that the deletion in strain $\mathrm{K} \Delta \mathrm{I} 8$ extends from the aroP gene through the ace operon and probably into the lpd gene (Table 4) unless it prevents lpd expression by some other mechanism. Immunological studies with antiserum raised against purified $E$. coli lipoamide dehydrogenase were used to test for the presence or absence of cross-reacting material (CRM) by immunodiffusion according to the methods described by Guest \& Creaghan (1974). No cross-reacting 
material could be detected in ultrasonic extracts of strain $\mathrm{K} \Delta \mathrm{I}$. This $\mathrm{CRM}^{-}$phenotype is also consistent with a deletion extending into the $l p d$ gene or one which prevents $l p d$ gene expression.

Other mutants. The unstable auxotrophic aroP mutants were also examined enzymologically by direct and complementation assays to elucidate further details of their phenotypes. It was concluded that strain $\mathbf{H} \Delta \mathbf{8}$ combines an nadC-aroP deletion with an aceF mutation and strains $\mathrm{H}_{7}, \mathrm{H} \Delta_{9}, \mathrm{H}_{\mathrm{II}} \mathrm{I}$ and $\mathrm{H}_{\mathrm{I}_{2}}$ have aroP mutations plus mutations in the ace $E$, ace $F$, $s u c B$ and $l p d$ genes, respectively (the $l p d$ mutation being $\mathrm{CRM}^{-}$). The data also suggested that all these strains, except $\mathrm{H} \Delta 7$, may have deletions in the aroP region which affect ace operon expression.

\section{DISCUSSION}

The results validated the prediction, based on the properties of two S. typhimurium strains (SMI6 and SM5I), that strains of $E$. coli deleted in aroP and adjacent genes may be found among spontaneous aroP mutants. Strains deleted for the nadC, aceE, ace $F$ and $l p d$ genes were isolated. The frequency of independent auxotrophic deletions amounted to $\mathrm{I} \cdot 2 \%$ of the spontaneous aroP mutants examined. This fell within the expected range of $\mathrm{I} \%$ to $5 \%$ for deletions among spontaneous mutants; a higher frequency would have been expected if non-auxotrophic aroP deletion mutants could have been detected and included. The use of a combination of aromatic amino-acid analogues presented a direct and fairly specific selection for aroP mutants (only $20 \%$ of the mutants from the first screening failed to exhibit the AroP- ${ }^{-}$phenotype in subsequent tests). Since the specific aromatic amino-acid transport systems have higher Michaelis constants than the general aromatic amino-acid permease (Brown, 1970; Thorne \& Corwin, 1975), it is probably essential to have the correct balance of analogues and concentrations sufficient to be inhibitory via the general (aroP) systems but not too high to afford entry via the specific permeases. The concentrations were critical and this may explain why the aroP mutants could still be retarded or inhibited when grown from small inocula.

The nutritionally-distinct classes within the group of 28 stable deletion strains, $\mathrm{Nad}^{-}, \mathrm{Nad}^{-}$ Ace $^{-}$, Ace $^{-}$, Lpd $^{-}$(including leaky 'Ace ${ }^{-}$' types), and further subdivisions based on the complement of functional $a c e E$ and $a c e F$ gene products, confirmed the gene order nadC-aroPaceE-aceF-lpd (Guest, 1974). The frequency with which different types were recovered did not appear to be strictly related to the length of genome deleted because the basic ( $\mathrm{Nad}^{-}, \mathrm{Ace}^{-}$ and $\left.\mathrm{Nad}^{-} \mathrm{Ace}^{-}\right)$types arose with almost equal frequency $(8$, Io and 9 respectively). However, only one strain $\left(\mathrm{K} \Delta_{\mathrm{I}} 8\right)$ with an aroP-lpd deletion, analogous to the deletion in $S$. typhimurium SMI6, was isolated and no strain with a deletion of the complete nadC-lpd region, corresponding to strain SM5I, was recovered. A further characteristic of the $S$. typhimurium strains, resistance to low concentrations of streptomycin, was not exhibited by any of the $E$. coli deletion strains, including strain $\mathrm{k} \Delta \mathrm{I} 8$. The $S$. typhimurium mutants also differed in that they did not require succinate for growth on glucose (Langley \& Guest, 1974). However, a succinate-independent partial revertant of strain $\mathrm{K} \Delta \mathrm{I} 8$, which was phenotypically more like strain SMI6, was still streptomycin-sensitive. The existence of viable deletions in the nadC-lpd region indicates that it is unlikely to contain other 'essential ' genes.

Of the 15 Ace $^{-}$deletions having no pyruvate dehydrogenase complex or EIp, only three had detectable E2p activity. Although this confirms the gene order aroP-aceE-aceF it cannot be concluded that all but three of these deletions end in the ace $F$ gene. The polarity of the ace operon is such that two-thirds of random deletions in the ace $E$ gene may be expected to generate reading-frame shifts affecting expression of the ace $F$ gene. 
Conclusions regarding the effects of the deletions on the control of ace and lpd gene expression must await a thorough genetic analysis. Nevertheless, the direct relationship between the lactate-resistance of pps mutants growing on acetate and the activity of the pyruvate dehydrogenase complex proved a useful criterion for evaluating the degree of expression of the ace operon. Constitutive synthesis of the complex produces resistance (Flatgaard et al., 197I) but none of the deletions generated resistance, e.g. by removing a gene specifying a negatively-acting repressor or by fusing the ace operon to a more efficient promoter. On the contrary, decreased resistance generated by the deletions in several Nadstrains and strains with 'Ace ${ }^{-}$'phenotypes corresponded to a lowering of the ace gene expression. Control elements between aroP and ace $E$ could again be affected by deletion or the ace operon could have been brought under the control of other, less effective, regulatory mechanisms. The leaky or 'Ace-' phenotype of several deletion mutants with decreased ace gene expression is noteworthy. The ability (albeit impaired) to grow on unsupplemented glucose and, to a lesser extent, succinate media, indicates that growth-limiting amounts of acetate are synthesized and that there is a greater demand for acetate during growth on succinate or that other sources of acetate (pyruvate oxidase or the phosphoroclastic reaction) are induced or derepressed to a greater extent on glucose. Point mutants with this 'Ace' ${ }^{-}$phenotype have not been reported. They may have been overlooked but could prove useful for studying the control of ace expression. The deletions generating the greatest lactate-sensitivity on acetate were totally deficient in pyruvate dehydrogenase complex activity as well as lacking phosphoenolpyruvate synthetase activity. In a $\mathrm{Pps}^{+}$background strains lacking pyruvate dehydrogenase complex activity, as well as $\mathrm{Ace}^{+}$strains, were unaffected by lactate. This suggests that phosphoenolpyruvate synthetase is primarily responsible for removing inhibitory pyruvate (formed from lactate) during growth on acetate plus lactate.

Only one strain, $\mathrm{k} \Delta \mathrm{I} 8$, lacked lipoamide dehydrogenase activity and this could be due to a deletion affecting either the lpd structural gene or its expression. All the deletion strains with Ace $^{-}$or ' $\mathrm{Ace}^{-}$' phenotype retained $\mathrm{E}_{3}$ activity at between $30 \%$ and $95 \%$ of the parental level. Expression of the lpd gene can thus be independent of the ace operon. The lower E3 activities of some deletion strains indicate that the ace operon may influence lipoamide dehydrogenase synthesis, e.g. by exerting polar or pseudo-polar effects on lpd gene expression at the level of transcription or translation. Alternatively, the deletions may have brought the lpd gene under the control of new regulatory elements which promote the observed levels of lipoamide dehydrogenase synthesis. However, similar effects on $\mathrm{E}_{3}$ synthesis have been observed with ace polar mutants; lpd expression was never less than $30 \%$ (Henning et al., I966; Creaghan \& Guest, 1972) and this is consistent with some form of ace-independent $l p d$ gene expression.

We wish to thank those who have provided the mutant strains used in this work, and D.L. acknowledges the receipt of a Research Studentship from the Science Research Council.

\section{REFERENCES}

Alwine, J. C., Russell, F. M. \& Murray, K. N. (1973). Characterization of an Escherichia coli mutant deficient in dihydrolipoyl dehydrogenase activity. Journal of Bacteriology 115, I-8.

AMEs, G. F. \& Roth, J. R. (1968). Histidine and aromatic permeases of Salmonella typhimurium. Journal of Bacteriology 96, $1742-1749$.

BRICE, C. B. \& KoRNBERG, H. L. (1967). Location of a gene specifying phosphopyruvate synthase activity on the genome of Escherichia coli K12. Proceedings of the Royal Society Br68, 281-292.

Brown, K. D. (1970). Formation of aromatic amino acid pools in Escherichia coli X-1 2. Journal of Bacteriology 104, $177-188$. 
Carrillo-Castaneda, G. \& ORTEga, M.V. (1970). Mutants of Salmonella typhimurium lacking phosphoenolpyruvate carboxykinase and $\alpha$-ketoglutarate dehydrogenase activities. Journal of Bacteriology ro2, 524530.

Creaghan, I. T. \& Guest, J. R. (1972). Amber mutants of the $\alpha$-ketoglutarate dehydrogenase gene of Escherichia coli KI 2. Journal of General Microbiology 71, 207-220.

Demerec, M. (1960). Frequency of deletions among spontaneous and induced mutations in Salmonella. Proceedings of the National Academy of Sciences of the United States of America 46, 1075-1079.

Flatgaard, J. E., Hoehn, B. \& HeNNING, U. (1971). Mutants of Escherichia coli k12 which synthesize the pyruvate dehydrogenase complex constitutively. Archives of Biochemistry and Biophysics 143, 461-470.

GuEst, J. R. (1974). Gene-protein relationships of the $\alpha$-keto acid dehydrogenase complexes of Escherichia coli $\mathrm{K} 12$ : chromosomal location of the lipoamide dehydrogenase gene. Journal of General Microbiology 80, 523-532.

Guest, J. R. \& Creaghan, I. T. (1972). Lipoamide dehydrogenase mutants of Escherichia coli K12. Biochemical Journal r3o, 8P.

Guest, J. R. \& Creaghan, I. T. (1973). Gene-protein relationships of the $\alpha$-keto acid dehydrogenase complexes of Escherichia coli $\mathrm{KI} 2$ : isolation and characterization of lipoamide dehydrogenase mutants. Journal of General Microbiology 5, 197-210.

Guest, J. R. \& CREAGHAN, I. T. (1974). Further properties of lipoamide dehydrogenase mutants of Escherichia coli KI2. Journal of General Microbiology 81, 237-245.

Gunsalus, I. C. \& Razzent, W. E. (1957). Preparation and assay of lipoic acid and derivatives. Methods in Enzymology 3, 94 I-946.

HAGER, L. P. \& KORNBerG, H. L. (196I). On the mechanism of $\alpha$-oxoglutarate oxidation in Escherichia coli. Biochemical Journal 78, 194-198.

Henning, U. \& Herz, C. (I964). Ein Strukturgen-Komplex für den Pyruvat-Dehydrogenase-Komplex von Escherichia coli K12. Zeitschrift für Vererbungslehre 95, 260-275.

Henning, U., Herz, C. \& Szolyvay, K. (1964). Polarisation und Disproportionalităt der Synthese von Enzymkomponenten des Pyruvat-Dehydrogenase-Komplexes als Mutationsfolge in Escherichia coli KI 2. Zeitschrift für Vererbungslehre 95, 236-259.

Henning, U., Dennert, G., Hertel, R. \& Shipp, W. S. (1966). Translation of the structural gene of the E. coli pyruvate dehydrogenase complex. Cold Spring Harbor Symposium for Quantitative Biology 3I, 227-234.

Henning, U., Dietrich, J., Murray, K. N. \& DePPE, G. (1968). Regulation of pyruvate dehydrogenase synthesis: substrate induction. In Molecular Genetics, pp. 223-236. Edited by H. G. Wittman and H. Schuster. Berlin: Springer-Verlag.

Henning, U., Busch, W., Deppe, G. \& Marek, R. (1969). Pyruvate dehydrogenase synthesis in E. coli KI 2. Federation of European Biochemical Societies Symposia 19, 19-28.

Herbert, A. A. \& Guest, J. R. (1969). Studies with $\alpha$-ketoglutarate dehydrogenase mutants of Escherichia coli. Molecular and General Genetics 105, 182-190.

Kornberg, H. L. (1966). The role and control of the glyoxylate cycle in Escherichia coli. Biochemical Journal 99, I-1 I.

Korngerg, H. L. \& SMITH, J. (1967). Genetic control of the uptake of pyruvate by Escherichia coli. Biochimica et biophysica acta 148, 59I-592.

LANGLEY, D. \& GUEST, J. R. (1974). Biochemical and genetic characteristics of deletion and other mutant strains of Salmonella typhimurium LT2 lacking $\alpha$-keto acid dehydrogenase complex activities. Journal of General Microbiology 82, 319-335.

LANGley, D. \& GuEsT, J. R. (1975). Deletion mutants of Escherichia coli KI 2 lacking $\alpha$-keto acid dehydrogenase complex activities. Proceedings of the Society for General Microbiology 2, 66-67.

LENNOX, E. S. (I955). Transduction of linked genetic characters of the host by bacteriophage PI. Virology I, 190-206.

LipmanN, F. \& Tuttue, L. C. (1945). A specific micromethod for the determination of acyl phosphates. Journal of Biological Chemistry r59, 2 I-28.

Lowry, O. H., Rosebrough, N. J., Farr, A. L. \& Randall, R. J. (1951). Protein measurement with the Folin phenol reagent. Journal of Biological Chemistry 193, 265-275.

Thorne, G. M. \& CoRWIN, L. M. (1975). Mutations affecting aromatic amino acid transport in Escherichia coli and Salmonella typhimurium. Journal of General Microbiology 90, 203-216.

Vogel, H. \& BonNer, D. M. (1956). A convenient growth medium for Escherichia coli and some other micro-organisms. Microbial Genetics Bulletin 13, 43-44.

Whlms, C. R., Oliver, R. M., Henney, H. R., MukHERJee, B. B. \& Reed, L. J. (1967). $\alpha$-Keto acid dehydrogenase complexes. VI. Dissociation and reconstitution of the dihydrolipoyl transacetylase of E. coli. Journal of Biological Chemistry 242, 889-897. 